\title{
Spinal Fluid Biomechanics and Imaging: An Update for Neuroradiologists
}

\author{
V. Haughton and K.-A. Mardal
}

\begin{abstract}
SUMMARY: Flow imaging with cardiac-gated phase-contrast MR has applications in the management of neurologic disorders. Together with computational fluid dynamics, phase-contrast MR has advanced our understanding of spinal CSF flow. Phase-contrast MR is used to evaluate patients with Chiari I malformation who are candidates for surgical treatment. In theory, abnormal CSF flow resulting from the abnormal tonsil position causes syringomyelia and other neurologic signs and symptoms in patients with Chiari I. CSF flow imaging also has research applications in syringomyelia and spinal stenosis. To optimize MR acquisition and interpretation, neuroradiologists must have familiarity with healthy and pathologic patterns of CSF flow. The purpose of this review is to update concepts of CSF flow that are important for the practice of flow imaging in the spine.
\end{abstract}

ABBREVIATION: PCMR $=$ phase-contrast MR

l: $\mathrm{n}$ the spinal canal, oscillatory CSF flow results primarily from the displacement of approximately $1.5 \mathrm{~mL}$ of fluid ${ }^{1}$ from the cranial cavity as intracranial blood vessels expand in arterial systole (Monro-Kellie doctrine). CSF in the spinal canal moves caudally when the systolic pulse wave reaches the brain and cephalad during diastole. Caudal CSF flow has greater velocities and shorter duration than cephalad flow (Fig 1). The fluid entering the spinal canal displaces blood from the epidural venous plexus in the spine. CSF flow and the venous displacement diminish progressively from the cephalic end of the cervical canal to the caudal end of the thoracic canal. CSF oscillations are coupled to CSF pressure oscillations that, in the healthy adult, are approximately $90^{\circ}$ out of phase with the velocity fluctuations. ${ }^{2}$ Elastic properties of the tissues surrounding the subarachnoid space theoretically induce pressure waves, which to date are not fully characterized. ${ }^{3}$ With contrast media or radionuclides in the spinal subarachnoid space, a slow convection of fluid is observed resulting from the continuous oscillation of CSF. ${ }^{4}$

Viscous and inertial properties, together with the complex anatomy of the subarachnoid space, determine CSF flow patterns. Viscosity creates a laminar pattern of flow, which can be thought of as layers of flowing fluid with no disruption between the layers.

From the Department of Radiology (V.H.), University of Wisconsin, Madison, Wisconsin; and Center for Biological Computing (K.-A.M.), Simula, Lysaker, Norway.

Please address correspondence to Victor Haughton, Department of Radiology, University of Wisconsin, 600 Highland Ave, Madison, WI 53792; e-mail:

haughtonvictor@gmail.com

- Indicates open access to non-subscribers at www.ajnr.org

http://dx.doi.org/10.3174/ajnr.A4023
Laminar CSF flow means that fluid moves with greater velocity in the center of a channel and with lesser velocity near a boundary resulting from frictional effects (Fig 2). Inertial forces resulting from the mass and velocity of CSF produce other effects in CSF flow. Acceleration and deceleration in CSF require the application of pressure. When a pressure gradient is applied to a laminar flow, the resulting velocities depend in part on the initial velocity of the fluid. Pressure gradients change flow velocities and affect flow

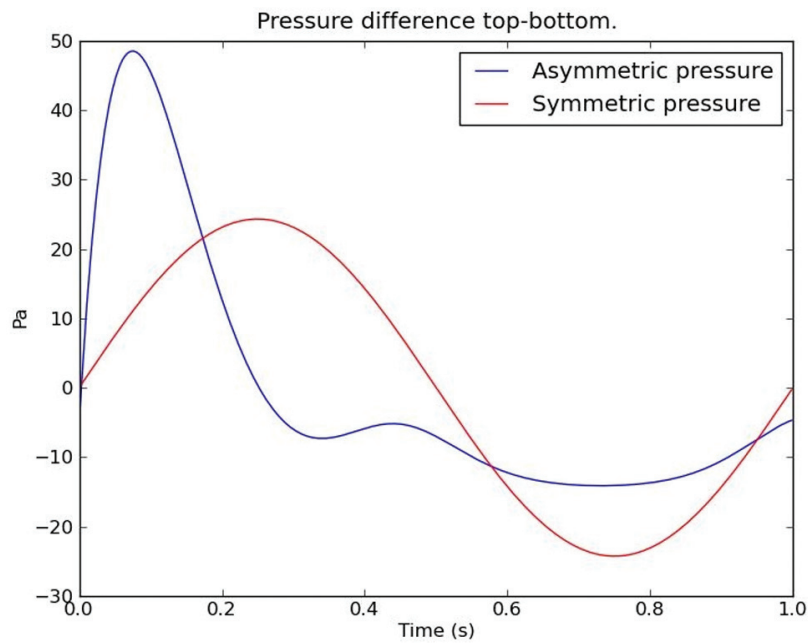

FIG 1. Plot of CSF velocity through the cardiac cycle (blue) and for comparison a sinusoid (red). CSF flow has greater velocity in the positive direction (systolic flow) than in the negative direction (diastolic flow). Systolic flow has shorter duration than diastolic flow. Flow volume in the 2 directions is equal. 


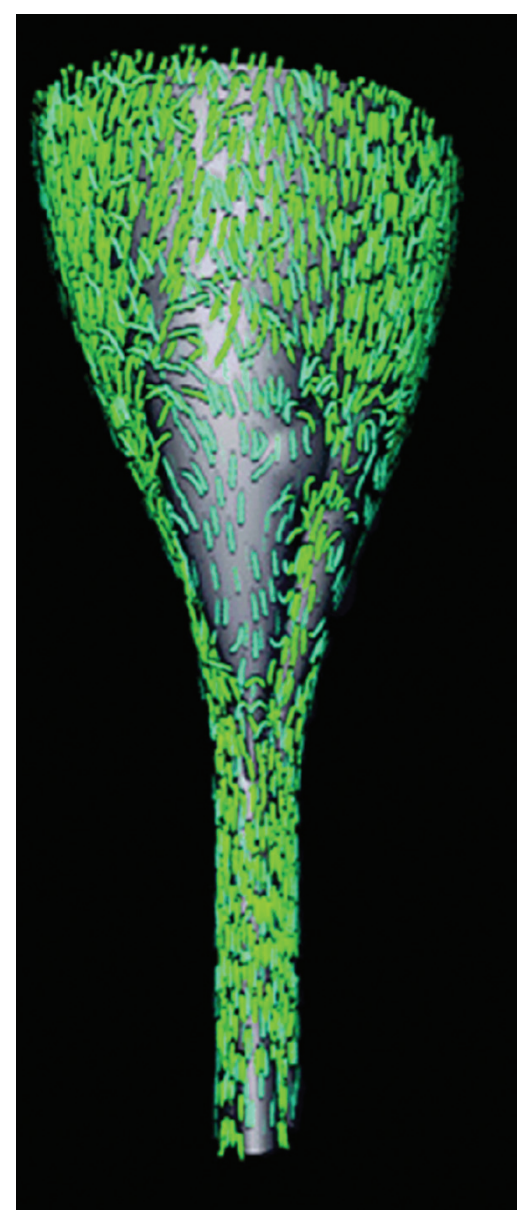

FIG 2. A 3D representation of CSF velocities in an idealized model of the posterior fossa and spinal canal of a patient with Chiari I. It displays the subarachnoid space posteriorly. It shows the direction and magnitude of flow throughout the space at one moment in the cardiac cycle by the direction and length of arrows. Flow velocities and flow directions vary from one region to another. The flow is more rapid at the cervical spine below the tonsils than at the craniovertebral junction. Flow predominates in the long axis of the model but has evidence of vortices in some areas.

near the dura and pia differently than flow in the middle of the channel. As flow oscillates between craniad and caudad directions, flow direction reverses throughout the subarachnoid space at slightly different times with the result that, for short periods of time, CSF flows in both craniad and caudad directions (synchronous bidirectional flow, Fig 3). ${ }^{5}$ This synchronous bidirectional flow does not appear in phase-contrast MR (PCMR) studies of healthy human subjects because of the limited temporal and spatial resolution of the MR method. Inertial properties of fluid may in some geometries create a pattern of flow called "flow separation," which results in eddy currents and vortices (Fig 4). ${ }^{6}$ Flow not aligned with the spinal axis can be demonstrated by computational fluid dynamics (Fig 5) ${ }^{7}$ and by PCMR when multiple gradient directions are used. ${ }^{6}$

The anatomy of the subarachnoid space increases the complexity of CSF flow patterns in the spine. The flow velocities in any axial section of the spine have nonuniform distributions throughout the cardiac cycle, as PCMR and flow simulations show (Figs 6 and 7). PCMR images demonstrate flow jets anterolateral to the spinal cord and lower velocities elsewhere. Flow patterns differ
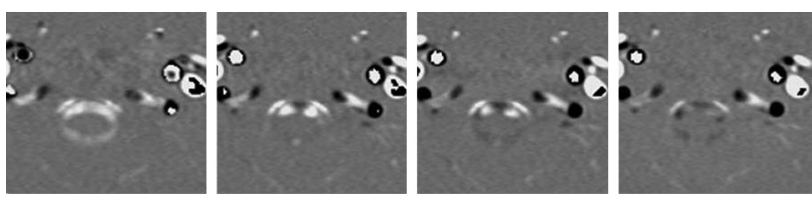

FIG 3. Four consecutive PCMR images of the 14 images obtained during one cardiac cycle in the upper cervical spine demonstrating flow in the subarachnoid space and in cervical arteries and veins. The first image shows flow anterior and posterior to the spinal cord has a positive sign (caudad direction). The second image shows flow anterolateral to the cord remains positive whereas flow in the midline anterior to the cord has a negative sign (cephalad). The third image shows more voxels with cephalad flow and fewer with caudad flow. The fourth image shows predominantly cephalad flow with little caudad flow detected. The 2 vessels anterolateral to the subarachnoid space have caudad flow in each of the 4 images.

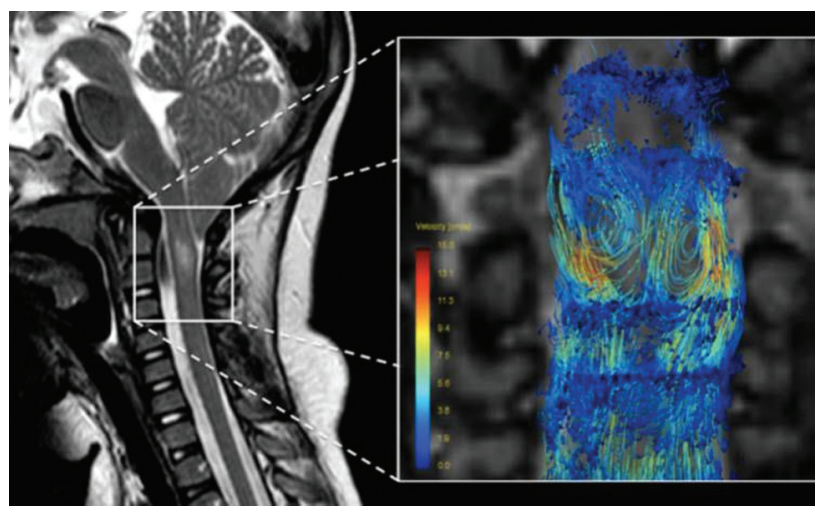

FIG 4. Sagittal T2-weighted MR image and coronal slab obtained with 4D PCMR acquisition method. The streamlines in PCMR image shows some flow along the spinal axis and some flow in vortices. Reproduced with permission from: Bunck AC, Kröger JR, Jüttner A, et al. Magnetic resonance 4D flow characteristics of cerebrospinal fluid at the craniocervical junction and the cervical spinal canal. Eur Radiol 2011;21:1788-96.

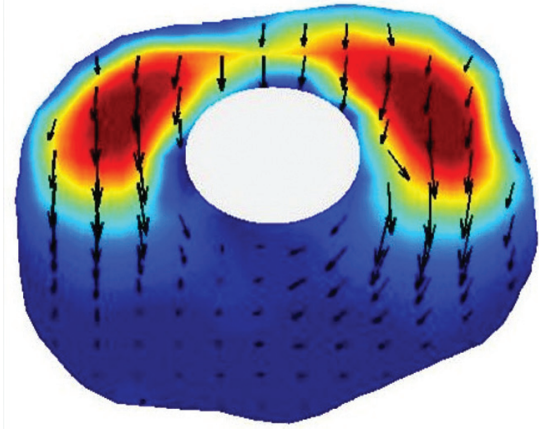

FIG 5. Computer graphic from a flow simulation showing flow along the spinal axis by red-blue-green color coding and in-plane flow by arrows in which velocity is coded by the dimensions of the arrows. Longitudinal flow predominates anterolateral to the cord (top of image) and in-plane flow predominates in a posterior direction. Modified from Roldan et al. ${ }^{7}$

from one spinal level to another. Peak CSF velocities increase progressively from $\mathrm{C} 1$ to $\mathrm{C} 4$ due to the tapering of this portion of the spinal canal. Systolic CSF velocities normally range up to 5 $\mathrm{cm} / \mathrm{s}$ in the foramen magnum and up to $10 \mathrm{~cm} / \mathrm{s}$ at $\mathrm{C} 4$ (Fig 8). ${ }^{8}$ Diastolic CSF velocities also vary with level. Simulations in patient-specific models show the progressive increase in systolic and 

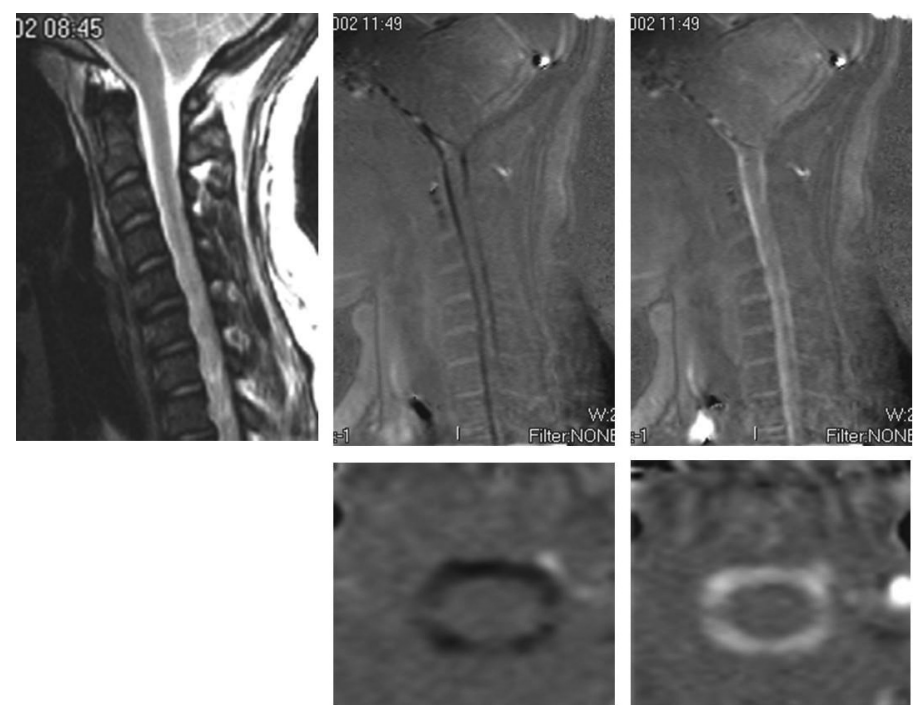

FIG 6. PCMR images in sagittal (upper row) and axial (lower row) views showing flow in one systolic and one diastolic phase of the cycle and for reference (top left) the sagittal T2-weighted image in the patient. These demonstrate more flow anterior to the cord than posterior. On careful inspection, the axial images show more flow anterolateral to the cord than posterior to it. Reproduced with permission from Hofkes SK, Iskandar BJ, Turski PA, et al. Differentiation between symptomatic Chiari I malformation and asymptomatic tonsilar ectopia by using cerebrospinal fluid flow imaging: initial estimate of imaging accuracy. Radiology 2007;245:532-40.

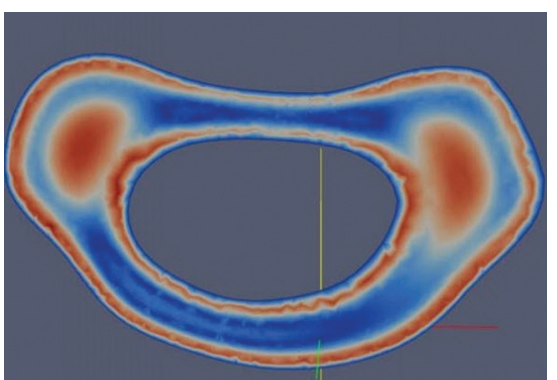

FIG 7. Graphic illustrating simulated flow in a patient-specific model of the subarachnoid space to illustrate the inhomogeneity of CSF flow. Flow velocity, in red-blue-green color coding, shows the greatest velocities anterolateral to the spinal cord. Modified from Rutkowska et al. ${ }^{9}$

diastolic velocities between the upper and midcervical spine (Fig 9). ${ }^{9}$

In fluid mechanics, the relative effects of inertia and viscosity on fluid can be inferred from the Reynolds and Womersley numbers. The Reynolds number, the ratio of inertial to viscous forces, differs little between CSF and blood in large arteries. The Womersley number, which quantifies the transient inertial forces in proportion to viscous effects, has a slightly lower value for CSF than for blood in large arteries. On the basis of the Reynolds and Womersley numbers, the oscillating spinal CSF flow has in theory some of the flow patterns observed in the arterial blood, though these are perhaps more complex because the surface has a more complex geometry.

Velocity fluctuations in the spinal fluid are associated with pressure fluctuations (Fig 10). In the healthy adult, spinal velocity and pressure fluctuations have an approximately $90^{\circ}$ phase difference. In the presence of obstruction, the phase difference narrows. ${ }^{2}$ Pressure fluctuations in the CSF produce transient pressure waves and gradients in the spinal cord. In portions of the spinal cord with a persistent central canal, pressure on the external surface of the cord may produce radial pressure gradients in the cord. ${ }^{10}$ In computational models of the spinal cord with realistic anatomic properties such as the central canal, the anterior median fissure, and white matter with anisotropic properties, pressure waves produce complex patterns of fluid movement in the cord. ${ }^{11}$ Further studies may clarify the role of these fluid movements in the pathogenesis of syringomyelia.

\section{CSF Flow in Different Physiologic \\ States}

Age, heart rate, blood pressure, intrathoracic pressure, and other physiologic parameters may factor in CSF flow patterns. The cervical spinal canal tends to taper more steeply in adults than in children. ${ }^{12}$ Children have greater CSF velocities than adults; this is likely because of a greater expansion of cerebral blood vessels during systole and age-related tapering of the cervical spinal canal. ${ }^{13}$ Physical exertion, which may trigger symptoms in patients with Chiari I, may hypothetically produce clinically important changes in CSF flow. Because of methodologic limitations, studies of CSF flow under nonresting physiologic conditions are infrequent. In CSF flow simulations in idealized models of a patient with Chiari and a healthy adult, increasing the pulse rate decreases the duration of the diastolic phase of CSF flow, increases the peak diastolic velocity, increases the magnitude of synchronous bidirectional flow, and doubles the pressure load on the spinal cord. ${ }^{14}$ In vivo, Bhadelia et $\mathrm{al}^{15,16}$ used a pencil beam MR technique to show that the Valsalva maneuver transiently reduces CSF velocity. Cough raises pressures in the CSF, to a greater extent in patients with Chiari I than in healthy controls. ${ }^{17}$ Williams ${ }^{18}$ used manometry in vivo to show that a Valsalva maneuver slows the passage of a pressure wave from the cranial vault to the lumbar spinal CSF. More studies are needed to understand the effect of physiologic factors on CSF flow.

\section{CSF Flow in the Chiari I Malformation}

Patients with Chiari commonly develop syringomyelia. The ectopic cerebellar tonsils in the upper cervical spinal canal in patients with Chiari I increase the complexity of flow patterns, the peak CSF velocities, and the magnitude of synchronous bidirectional flow. Flow velocities in the foramen magnum reach $12 \mathrm{~cm} / \mathrm{s}$ in patients with Chiari $\mathrm{I}^{19,20}$ or more ${ }^{6}$ compared with $5 \mathrm{~cm} / \mathrm{s}$ in healthy adults. ${ }^{20}$ Both peak systolic and diastolic velocities are elevated in Chiari I. In Chiari I, CSF velocities along the spinal axis are greater than in healthy subjects when subjected to the same pressure drop. PCMR demonstrates greater complexity of flow with larger jets and increased flow inhomogeneity in patients with Chiari I ${ }^{19}$ (Fig 9). Simulations of CSF flow in patient-specific models of the subarachnoid spaces in patients with Chiari 


\section{Velocities and PC Images by Region in a Chiari I Patient}

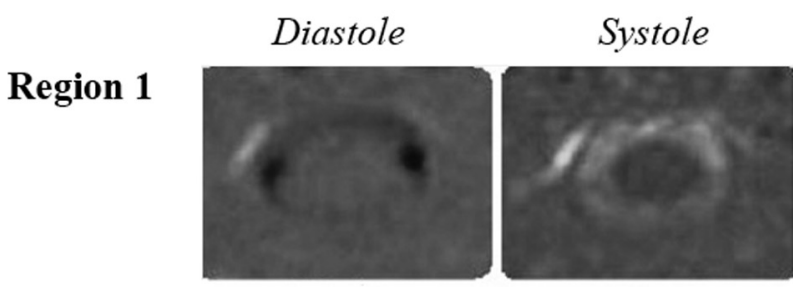

\section{Region 2}
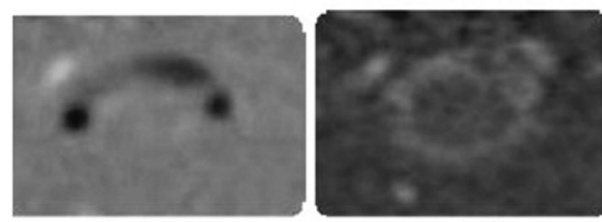

\section{Region 3}
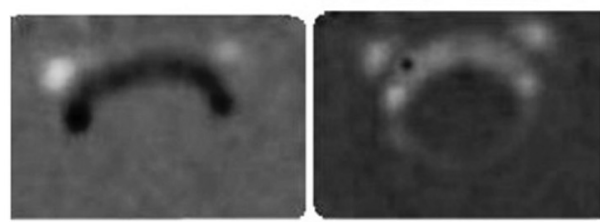

\section{Region 4}
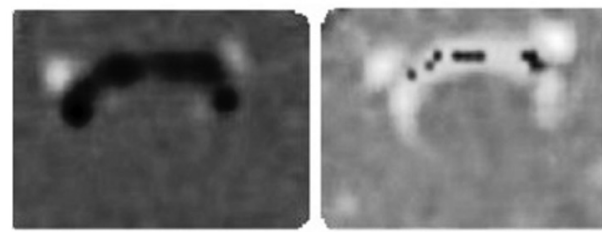

FIG 8. PCMR images of diastolic flow (left column) and systolic flow (right column) in successively lower levels of the upper cervical spinal canal in a patient with Chiari I. CSF velocities increase from region 1 near the tonsils to region 4 near the $\mathrm{C} 4$ level. At $\mathrm{C} 4$, aliasing produces negative flow voxels (black) scattered among the voxels with positive flow (white voxels). Modified from Shah et al. ${ }^{8}$
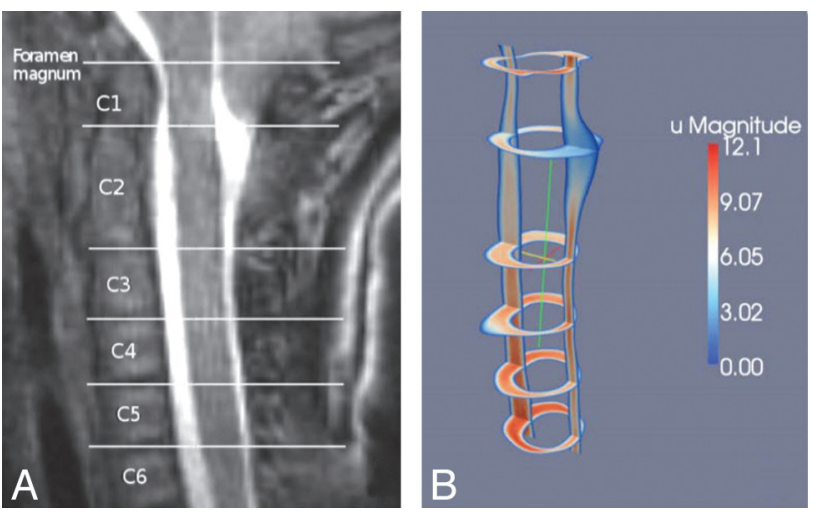

FIG 9. Sagittal T2-weighted image in a patient with a Chiari I malformation and graphic showing simulated flow velocities in the same patient by sagittal and multiple axial sections. The flow simulations show inhomogeneous flow patterns, greatest flow anterolateral to the cord, and increasing velocities between $\mathrm{Cl}$ and $\mathrm{C} 4$. Reproduced from Rutkowska et al. ${ }^{9}$
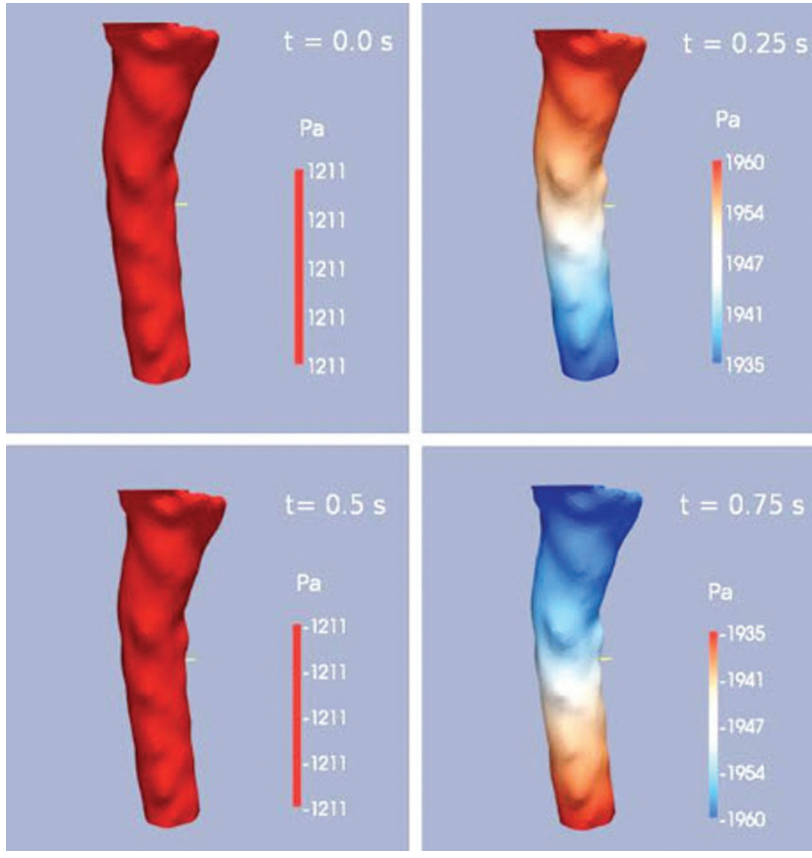

FIG 10. Graphic showing pressure gradients along the cervical spinal canal at 4 phases of the cardiac cycle. Pressure is coded by the redblue-green scale. At one phase, pressure decreases from $\mathrm{Cl}$ to $\mathrm{C}$; at one phase it increases from $\mathrm{Cl}$ to $\mathrm{C} 7$, and at 2 phases in the cycle, no gradient is present. The pressure oscillates between these pressure patterns during the cycle. Velocities peak approximately $90^{\circ}$ out of phase with the pressure in healthy adults. Modified from Roldan et al. ${ }^{7}$

document increased synchronous bidirectional flow. ${ }^{5,9}$ In symptomatic patients with Chiari, PCMR demonstrates flow simultaneously in a caudad and cephalad direction (synchronous bidirectional flow) for $15-20 \%$ of the cardiac cycle, that is 2 or 3 phases in a typical 14-phase PCMR acquisition ${ }^{19}$ (Fig 3) and not in asymptomatic patients with Chiari or controls. ${ }^{21}$ Other differences between patients with Chiari and controls were reported, including different durations of systole and shifts in phase between velocity and pressure. ${ }^{22}$ Although incompletely studied, transitional or turbulent CSF flow may occur in patients with Chiari I. Simulations in a model of a typical patient with Chiari I showed flow instabilities that may develop into turbulent flow though did not show self-sustained turbulence. ${ }^{23}$

The clinical objective of most PCMR imaging is to help distinguish patients who are symptomatic from the Chiari malformation from those whose symptoms are not related to their ectopic tonsil position. Readers interpreting PCMR images have an accuracy of $60-70 \%$ in differentiating symptomatic from asymptomatic cases. ${ }^{21}$ Symptomatic cases of Chiari I typically have evidence of synchronous bidirectional flow and asymptomatic cases do not.

Cervical spinal canal dimensions, which affect local CSF velocities, differ between patients with Chiari and controls. The cervical spinal canal tapers less steeply in patients with Chiari with the result that peak CSF velocities, though more elevated in the foramen magnum, increase less rapidly than in healthy adults. ${ }^{12,24}$ Spinal canal tapering in patients with Chiari with a syrinx differs from those without a syrinx (A. Thompson, unpublished data, 2014). Tapering affects CSF pressures and pressure gradients in the upper cervical spinal canal. Pressures and pressure gradients 


\section{Pressure Gradient for the Chiari, Normal and Post-operative Models}

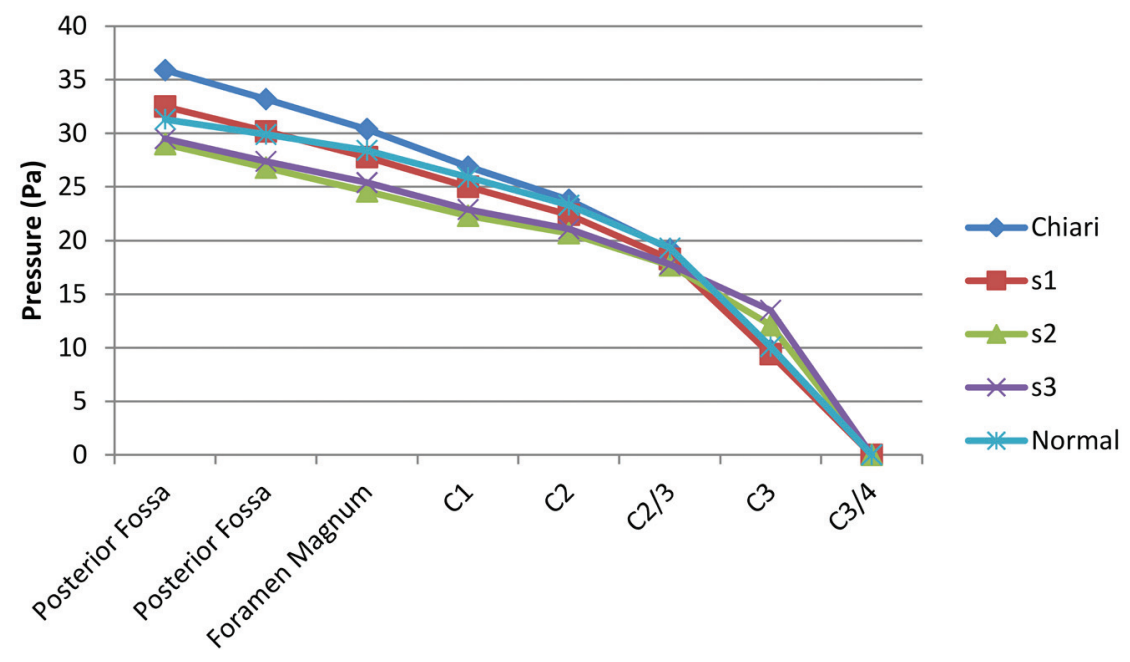

FIG 11. Plot of relative pressure against position from the midposterior fossa to the $C 3 / 4$ level in 5 computational models: an idealized healthy model, an idealized patient with Chiari I, and 3 models showing the Chiari model with various amounts of craniovertebral decompression. At this phase of the cycle, pressure decreases from posterior fossa to $\mathrm{C} 3 / 4$. The gradients are steepest in the Chiari I model and least steep in the healthy model. The 3 degrees of craniovertebral decompression appear to lower pressures in the foramen magnum and to normalize pressures at the $\mathrm{C} 3 / 4$ level.

increase in proportion to the tapering in patients with Chiari I without and with syrinx. The role of spinal canal tapering in the pathogenesis of syringomyelia requires more study.

Craniovertebral decompression and removal of a portion of the inferior posterior calvaria and the posterior part of $\mathrm{C} 1$ to modify CSF flow usually reverses the growth of syrinx and relieves neurologic signs and symptoms in the patient with Chiari I. This surgical procedure reduces CSF velocities in the foramen magnum but not to healthy levels. ${ }^{25}$ Simulations show that a craniovertebral surgical defect normalizes velocities in the midcervical spine and reduces them in the upper cervical spine $^{26}$ (Fig 11). It also lowers the pressures to which the spinal cord is subjected. Determining how the changes in pressure gradients lead to resolution of a syrinx requires additional simulations.

\section{CSF Flow in Other Spinal Pathologies}

CSF flow dynamics in idiopathic syringomyelia, arachnoiditis, and cervical spinal stenosis have been investigated.

PCMR imaging shows hyper kinetic flow in idiopathic syringomyelia. ${ }^{27}$ This suggests that pathogenetic mechanisms in the condition may be similar to those in Chiari I. The term Chiari 0 is sometimes used to describe idiopathic syringomyelia because idiopathic syringomyelia and Chiari I have similar symptoms and signs except for tonsilar ectopia. Like a Chiari I malformation, idiopathic syringomyelia has smaller than normal posterior fossa dimensions. ${ }^{28}$ The spine in patients with idiopathic syringomyelia tapers more like patients with Chiari I with syrinx than patients with Chiari I without syrinx or healthy adults (A. Struck, unpublished data, 2014).

Arachnoiditis, characterized by thickening and stiffening of the membrane lining the subarachnoid space has, in CSF flow simulations, higher than normal pressure pulses. ${ }^{29}$ Investigators hypothesize that elevated CSF pressure may be a significant factor in the pathogenesis of syringomyelia that may accompany arachnoiditis.

Syringomyelia may occur below an obstruction of the cervical spinal canal secondary to osteochondrosis. ${ }^{30}$ Laminectomy at the level of the stenosis effectively treats the syrinx in these patients. ${ }^{31}$

\section{CONCLUSIONS}

Spinal CSF has complex oscillatory flow patterns resulting from the displacement of cranial CSF. MR flow imaging shows cyclic changes in spinal fluid flow related to the cardiac cycle, spinal fluid flow jets related to the complex spinal anatomy, and flow vortices. It shows hyperdynamic CSF flow in the presence of tonsilar ectopia. Simulations show these complex flow patterns and provide measurements of the CSF pressure gradients through the cardiac cycle. Engineering calculations suggest that the inertial and viscous forces in CSF have similar proportions to blood flowing in the aorta. Ongoing studies suggest how CSF flow may have a role in the development of syringomyelia and how surgical management may be optimized.

\section{REFERENCES}

1. Gupta S, Soellinger M, Grzybowski DM, et al. Cerebrospinal fluid dynamics in the human cranial subarachnoid space: an overlooked mediator of cerebral disease. I. Computational model. J R Soc Interface 2010;7:1195-204

2. Støverud KH, Langtangen HP, Haughton V, et al. CSF pressure and velocity in obstructions of the subarachnoid spaces. Neuroradiol $J$ 2013;26:218-26

3. Shaffer N, Martin BA, Rocque B, et al. Cerebrospinal fluid flow impedance is elevated in Type I Chiari malformation. J Biomech Eng 2014;136:021012

4. Greitz D, Hannerz JA. Proposed model of cerebrospinal fluid circulation: observations with radionuclide cisternography. AJNR Am J Neuroradiol 1996;17:431-38

5. Linge SO, Haughton V, Løvgren AE, et al. Effect of tonsillar herniation on cyclic CSF flow studied with computational flow analysis. AJNR Am J Neuroradiol 2011;32:1474-81

6. Bunck AC, Kroeger JR, Juettner A, et al. Magnetic resonance 4D flow analysis of cerebrospinal fluid dynamics in Chiari I malformation with and without syringomyelia. Eur Radiol 2012;22:1860-70

7. Roldan A, Wieben $\mathrm{O}$, Haughton V, et al. Characterization of CSF hydrodynamics in the presence and absence of tonsillar ectopia by computational flow analysis. AJNR Am J Neuroradiol 2009;30: 941-46

8. Shah S, Haughton V, del Río AM. CSF flow through the upper cervical spinal canal in Chiari I malformation. AJNR Am J Neuroradiol 2011;32:1149-53

9. Rutkowska G, Haughton V, Linge S, et al. Patient-specific 3D simulation of cyclic CSF flow at the craniocervical region. AJNR Am J Neuroradiol 2012;33:1756-62 
10. Drøsdal IN, Mardal K-A, Støverud K, et al. Effect of the central canal in the spinal cord on fluid movement within the cord. Neuroradiol $J$ 2013;26:585-90

11. Støverud K. Relation between the Chiari I malformation and syringomyelia from a mechanical perspective. http://folk.uio.no/kentand/thesis-5.pdf. Accessed May 18, 2014

12. Hammersley J, Haughton V, Wang Y, et al. Tapering of the cervical spinal canal in patients with scoliosis with and without the Chiari I malformation. AJNR Am J Neuroradiol 2012;33:1752-55

13. Iskandar BJ, Haughton $V$. Age-related variations in peak cerebrospinal fluid velocities in the foramen magnum. J Neurosurg 2005;103(6 suppl):508-11

14. Linge SO, Mardal K-A, Haughton V, et al. Simulating CSF flow dynamics in the normal and the Chiari I subarachnoid space during rest and exertion. AJNR Am J Neuroradiol 2013; 34:41-45

15. Bhadelia RA, Frederick E, Patz S, et al. Cough-associated headache in patients with Chiari I malformation: CSF flow analysis by means of cine phase-contrast MR imaging. AJNR Am J Neuroradiol 2011;32: $739-42$

16. Bhadelia RA, Madan N, Zhao Y, et al. Physiology-based MR imaging assessment of CSF flow at the foramen magnum with a Valsalva maneuver. AJNR Am J Neuroradiol 2013;34:1857-62

17. Sansur CA, Heiss JD, DeVroom HL, et al. Pathophysiology of headache associated with cough in patients with Chiari I malformation. J Neurosurg 2003;98:453-58

18. Williams B. Cerebrospinal fluid pressure changes in response to coughing. Brain 1976;99:331-46

19. Quigley MF, Iskandar B, Quigley ME, et al. Cerebrospinal fluid flow in foramen magnum: temporal and spatial patterns at MR imaging in volunteers and in patients with Chiari I malformation. Radiology 2004;232:229-36

20. Hofmann E, Warmuth-Metz M, Bendszus M, et al. Phase-contrast MR imaging of the cervical CSF and spinal cord: volumetric motion analysis in patients with Chiari I malformation. AJNR Am J Neuroradiol 2000;21:151-58

21. Hofkes SK, Iskandar BJ, Turski PA, et al. Differentiation between symptomatic Chiari I malformation and asymptomatic tonsilar ectopia by using cerebrospinal fluid flow imaging: initial estimate of imaging accuracy. Radiology 2007;245:532-40

22. Stoodley Bilston LE, Stoodley MA, Fletcher DF. The influence of the relative timing of arterial and subarachnoid space pulse waves on spinal perivascular cerebrospinal fluid flow as a possible factor in syrinx development. J Neurosurg 2010;112:808-13

23. Helgeland A, Mardal K-A, Haughton V, et al. Numerical simulations of the pulsating flow of cerebrospinal fluid flow in the cervical spinal canal of a Chiari patient. J Biomech 2014;47:1082-90

24. Hirano M, Haughton V, Munoz del Rio A. Tapering of the cervical spinal canal in patients with Chiari I malformations. AJNR Am J Neuroradiol 2012;33:1326-30

25. Dolar MT, Haughton VM, Iskandar BJ, et al. Effect of craniocervical decompression on peak CSF velocities in symptomatic patients with Chiari I malformation. AJNR Am J Neuroradiol 2004;25:142-45

26. Linge SO, Mardal KA, Helgeland A, et al. Effect of craniovertebral decompression on CSF dynamics in Chiari malformation Type I studied with computational fluid dynamics. J Neurosurg Spine 2014; Aug 1. [Epub ahead of print]

27. Struck AF, Haughton VM. Idiopathic syringomyelia: phase-contrast MR of cerebrospinal fluid flow dynamics at level of foramen magnum. Radiology 2009;253:184-90

28. Bogdanov EI, Heiss JD, Mendelevich EG, et al. Clinical and neuroimaging features of "idiopathic" syringomyelia. Neurology 2004;62: 791-94

29. Cheng S, Stoodley MA, Wong J, et al. The presence of arachnoiditis affects the characteristics of CSF flow in the spinal subarachnoid space: a modelling study. J Biomech 2012;45:1186-91

30. Lee JH, Chung CK, Kim HJ. Decompression of the spinal subarachnoid space as a solution for syringomyelia without Chiari malformation. Spinal Cord 2002;40:501-06

31. Kato N, Tanaka T, Nagashima H, et al. Syrinx disappearance following laminoplasty in cervical canal stenosis associated with Chiari malformation-case report. Neurol Med Chir (Tokyo) 2010;50: 172-74 